\title{
EFFECTIVENESS OF LIFESTYLE MODIFICATION ON WEIGHTLOSS AND QUALITY OF LIFE IN OBESE WOMEN WITH POLYCYSTIC OVARIAN SYNDROME
}

\author{
VINAYA RAJENDRA PATIL ${ }^{1 *}$, POOVISHNU DEVI THANGAVELU², VAISHALI KRISHNAT JAGTAP ${ }^{3}$ \\ ${ }^{1}$ Department of Community , Faculty of Physiotherapy, Krishna University, Karad, Maharashtra, India. ${ }^{2}$ Department of Cardio Respiratory, \\ Faculty of Physiotherapy, Krishna University, Karad, Maharashtra, India. ${ }^{3}$ Department of Musculoskeletal, Faculty of Physiotherapy, \\ Krishna University, Karad, Maharashtra, India. Email: patil.vinaya1993@gmail.com
}

Received: 01 March 2018, Revised and Accepted: 31 May 2018

ABSTRACT

Objectives: (1) The objectives of this study were to determine the effectiveness of lifestyle modification on weight loss and the quality of life in obese women with the polycystic ovarian syndrome and (2) to determine the effectiveness of conventional physiotherapy on weight loss and the quality of life in obese women with polycystic ovarian syndrome.

Methods: Ethical clearance was obtained from the Institutional Ethical Committee. A total of 40 obese women with the polycystic ovarian syndrome (PCOS) were selected and divided into two groups, Group A (N=17) received conventional physiotherapy alone, and Group B (n=15) received lifestyle modification along with conventional physiotherapy. The preassessment of body weight is measured by body mass index (BMI); the waist-hip ratio (W-H ratio) and body fat percentage and the quality of life were scored as per the PCOS questionnaire (PCOSQ); and postinterventional assessment was taken for the same after 9 months.

Result: Intergroup statistical analysis for BMI revealed extremely significant in postintervention for Group B (P<0.0001). W-H ratio and PCOSQ were extremely significant for Group B ( $<0.0001)$. While postintervention analysis showed extremely significant difference between Group A and Group B $(\mathrm{P}<0.0001)$. Group B treated with lifestyle modification, and conventional physiotherapy was extremely significant.

Conclusion: Lifestyle modification with conventional physiotherapy helped in reducing weight and showed the increased quality of life in women with PCOS.

Keywords: Lifestyle modification, Polycystic ovarian syndrome, Aerobic, Strengthening, Obese, Body mass index.

(C) 2018 The Authors. Published by Innovare Academic Sciences Pvt Ltd. This is an open access article under the CC BY license (http://creativecommons. org/licenses/by/4. 0/) DOI: http://dx.doi.org/10.22159/ajpcr.2018.v11i9.25575

\section{INTRODUCTION}

The polycystic ovarian syndrome (PCOS) is the most common endocrine disorder which affects about $5-10 \%$ of women of reproductive age [1]. Presentation of PCOS includes menstrual irregularity, infertility, hirsutism, and pregnancy complication [1,2]. Key components in the pathogenesis of PCOS deal between insulin resistance and hyperandrogenism [2]. Women with PCOS are more likely to be overweight [2,3]. Some study suggests that prevalence of obese women is higher in PCOS women as compared with healthy controls [4].

PCOS women have an increased risk of metabolic disorder, Type 2 diabetics, impaired glucose tolerance, and cardiovascular diseases [1,2,5]. Insulin resistance is important factor in the pathophysiology of PCOS women [6]. Hirsutism (excess body hair) leads significant psychological stress also infertility leads to tension within the family and altered self-perception [7,8]. Psychological problem includes anxiety and poor self-esteem [1].

It is estimated that about $40-60 \%$ of PCOS women are obese or overweight with greater abdominal or visceral adiposity [1]. The presentation of obese PCOS women shows central obesity [4].

About 5\% of weight loss show reduced insulin level, improve ovulatory functions, and also reduce serum testosterone [2]. Considering up to $80 \%$ of individuals who reduce at least $5-7 \%$ of weight loss show improvement in ovulation and menstrual cyclicity with metabolic improvements where lifestyle treatment should be the first-line treatment for PCOS and remains supplementary when additional treatment is introduced $[4,9,10]$. Obesity and insulin resistance play an important role in the pathogenesis of PCOS [4]. This indirectly suggests that treatment that reduces body weight and increases insulin insensitivity can be beneficial for obese.

Lifestyle modification is often regarded as the first-line treatment for PCOS women [4]. Weight loss through lifestyle modification, which includes dietary modification, physical activity, and behavioral changes; medication improves insulin resistance, reduces hyperandrogenism, and eases the clinical severity of PCOS [4]. Therefore, lifestyle modification is the first line of management in PCOS for the prevention of weight gain [3]. Research studies have reported that lifestyle treatment lasting for 4-6 months shows improvement in reproductive functions [4]. The proportion of PCOS women who underwent lifestyle management showed significant improvements in menstrual cyclicity or ovulation which was $44 \%-57 \%$ [4]. The lifestyle modification programs with diet and physical activity are recommended to delay the onset of diabetic type 2, one of the serious complications of PCOS [4].

\section{METHODS}

Ethical clearance was obtained from the Institutional Ethical Committee, KIMSDU, karad. This study included 40 obese women diagnosed with PCOS who were divided into two groups. The study protocol was approved by the Regional Ethical Committee. Exclusion criteria included Type II diabetes, symptomatic cardiovascular problems, any concurrent hormone therapy within 6 weeks, practicing any type of weight loss program, and pregnant women's or who are willing to conceive. After baseline assessment, which included demographic data, body mass index (BMI), W-H ratio, questionnaire PCOS (PCOSQ), and body fat percentage they were allocated to respective groups using random sampling method. 
The first group (Group A n=17) received conventional physiotherapy with medication. The second group (Group $\mathrm{B}=15$ ) received lifestyle modification with conventional physiotherapy and medication (Fig. 1).

\section{Sampling method}

Group A was given following exercises.

\section{Structured exercise program for Group B}

A. Warm up and cool down exercises: Stretching of quadriceps, calf, and triceps and hamstring. Furthermore, in sanding basic exercises such as spread leg bending, spread-leg twisted bending, and spread leg forward bending.

B. Strengthening program in PCOS: The primary etiology of PCOS is insulin resistant. While skeletal muscles are the largest reservoir for insulin action. Strengthening program helps to reduce muscle fat [11]. Strengthening program includes push up on wall squats, oblique curls, bird dog, abdominal, hallowing, bilateral straight leg raise with head raise, dead bug, incline push-ups, and forward lunges for each 10 repetitions. Strengthening muscles give ability to perform everyday activities lead and boost metabolic rate which indeed burns more calories even when your body is at rest.

C. Diet: 1200-1500 calorie/day energy restriction is required for weight loss. High-protein diet is a part, which includes egg white, beans, and pulses. Green tea, idli, and oil-free food should be included. Fruits such as apple, guava, pear, grapes, oranges sweet-lime, etc., should be included. A PCOS friendly food includes tomatoes, sweet potatoes, lentils, pumpkin, and almonds walnuts. Oil-free roti avoid food with high-glycemic index such as bakery and dairy products. Leafy vegetables and high-fiber foods are included in the study. Whereas, junk food and dairy products are total prohibited instead skimmed milk can be a part of their diet. Pulses play an important role in weight control [12]. A diabetic diet promotes weight loss [13]. The best diet type is a low-glycemic index diet [13].

D. Behavioral management: Motivation to lose weight and gain good body image, to set goal and have confidence about self-body when around the people. Cognitive behavioral therapy, to avoid negative thoughts and to improve the state of mind.

Group A was given the following:

A. Exercises: Walking as warm and cool down each 10 min and brisk walking for $15 \mathrm{~min}$

\section{RESULTS}

1. BMI

In the present study, preinterventional means of BMI score was $30.89 \pm 0.8$ in Group B, and 30.78 \pm 1.01 in Group A whereas postinterventional mean was $28.7 \pm 1.13$ for BMI in Group B, and $30.86 \pm 0.52$ in Group A, respectively.

Intergroup analysis of BMI score was done using unpaired $t$-test. Postintervention analysis showed extremely significant difference between Group A and Group B ( $\mathrm{p}=0.0002$ ) (Table 1 and Fig. 2)

2. $\mathrm{W}$-H ratio

In the present study, preinterventional mean was $0.81 \pm 0.3$ in Group A, and $0.82 \pm 0.02$ in Group B whereas postinterventional mean was $0.71 \pm 0.02$ in Group B, and $0.79 \pm 0.03$ in Group A, respectively. Intergroup analysis of $\mathrm{W}$-Hip ratio was done using unpaired $t$-test, postintervention analysis showed extremely significant difference between depression and anxiety component of Group A and B ( $\mathrm{p} \leq 0.0001$ ), respectively (Table 2 and Fig. 3 ).

3. PCOSQ

In the present study, preinterventional mean PCOSQ score was $131.1 \pm 15.04$ in Group A, and 115.4 \pm 12.08 in Group B ( $t=3.226)$ whereas postinterventional mean PCOSQ score was $148.05 \pm 12.1$ in Group B, and 128.8 \pm 15.2 in Group A ( $t=5.420)$, respectively. Intragroup analysis of PCOSQ score was done using unpaired $t$-test. Postintervention analysis showed extremely significant difference between PCOSQ in Group A and B $(<0.0001)$, respectively (Table 3 and Fig. 4).

4. Body fat
Table 1: Comparison between pre and post-BMI mean values between groups

\begin{tabular}{|c|c|c|c|c|c|}
\hline \multirow[t]{2}{*}{ Group } & \multicolumn{2}{|c|}{ Pretreatment } & \multicolumn{2}{|c|}{ Posttreatment } & \multirow[t]{2}{*}{$\mathbf{p}$} \\
\hline & Mean \pm SD & Median & Mean \pm SD & Median & \\
\hline A & $30.78 \pm 1.01$ & 30.5 & $30.86 \pm 0.52$ & 29.8 & 0.0042 \\
\hline B & $30.89 \pm 0.8$ & 30.2 & $28.7 \pm 1.13$ & 28.5 & $<0.0001^{* * *}$ \\
\hline
\end{tabular}

Table 2: Comparison between pre and postmean values of waist-hip ratio between groups

\begin{tabular}{|c|c|c|c|c|c|c|}
\hline \multirow[t]{2}{*}{ Group } & \multicolumn{2}{|c|}{ Pretreatment } & \multicolumn{2}{|c|}{ Posttreatment } & \multirow[t]{2}{*}{$\mathbf{t}$} & \multirow[t]{2}{*}{$\mathbf{p}$} \\
\hline & Mean \pm SD & Median & Mean \pm SD & Median & & \\
\hline A & $0.81 \pm 0.3$ & 0.82 & $0.79 \pm 0.03$ & 0.8 & 16.62 & 0.0003 \\
\hline B & $0.82 \pm 0.02$ & 0.82 & $0.71 \pm 0.02$ & 0.71 & 4.861 & $<0.0001$ \\
\hline
\end{tabular}

Table 3: Comparison between pre and postmean values of PCOSQ scores between groups

\begin{tabular}{lllll}
\hline \multirow{2}{*}{ Group } & Pretreatment & & Posttreatment & \multirow{2}{*}{$\mathbf{p}$} \\
\cline { 2 - 2 } & Mean \pm SD & & Mean \pm SD & \\
\hline A & $131.17 \pm 15.05$ & & $121.8 \pm 15.27$ & 0.0004 \\
B & $115.46 \pm 12.08$ & & $148.05 \pm 12.1$ & $<0.0001$ \\
\hline$* * *$ Extremely significant & & &
\end{tabular}

Table 4: Comparison between pre and postmean values of body fat ratio between groups

\begin{tabular}{|c|c|c|c|c|c|}
\hline \multirow[t]{2}{*}{ Group } & \multicolumn{2}{|c|}{ Pretreatment } & \multicolumn{2}{|c|}{ Posttreatment } & \multirow[t]{2}{*}{$\mathbf{p}$} \\
\hline & Mean \pm SD & Median & Mean \pm SD & Median & \\
\hline $\mathbf{A}$ & $31.17 \pm 1.9$ & 31 & $30.6 \pm 0.03$ & 31 & 0.0003 \\
\hline B & $32.2 \pm 1.97$ & 32 & $27.7 \pm 1.8$ & 28 & $<0.0001$ \\
\hline
\end{tabular}

Table 5: Post-post values of all outcome measures in between Group A and B

\begin{tabular}{lllllll}
\hline \multirow{2}{*}{$\begin{array}{l}\text { Outcome } \\
\text { measures }\end{array}$} & Mean \pm SD & & \multicolumn{2}{c}{ Median } & \multirow{2}{*}{ P value } \\
\cline { 2 - 3 } & Group A & Group B & & Group A & Group B & \\
\hline BMI & $30.8 \pm 0.52$ & $28.7 \pm 1.13$ & & 29.8 & 28.5 & $<0.0001$ \\
W-HIP & $0.79 \pm 0.03$ & $0.71 \pm 0.02$ & 0.8 & 0.71 & $<0.0001$ \\
PCOSQ & $121.8 \pm 15.2$ & $148.05 \pm 12.1$ & 120 & 150 & $<0.0001$ \\
Body-fat & $27.7 \pm 1.86$ & $30.66 \pm 2.12$ & 31 & 28 & $<0.0001$ \\
\hline
\end{tabular}

In the present study, preinterventional means of body fat score were 31.17 \pm 1.91 in Group A, and 32.2 \pm 1.97 in Group B whereas postinterventional means of body fat were $27.7 \pm 1.86$ in Group B, and 30.66 \pm 2.12 in Group A, respectively.

Intergroup analysis of BMI score was done using unpaired $t$-test. Preinterventional analysis showed no significant difference between Group A and B $(\mathrm{p}=0.0003)$. Postintervention analysis showed extremely significant difference between Group A and B ( $p=0.0001)$ (Table 4 and Fig. 5)

\section{DISCUSSION}

Exercises and adopting healthy lifestyle created a good body and mental image in the patient that drastically improved which directly showed impact on higher quality of life according to PCOSQ and also showed a significant improvement in other outcome measures.

Visceral fat itself secretes adipokines which impair insulin sensitivity in tissue such as liver and skeletal muscle, insulin resistance is manifested 


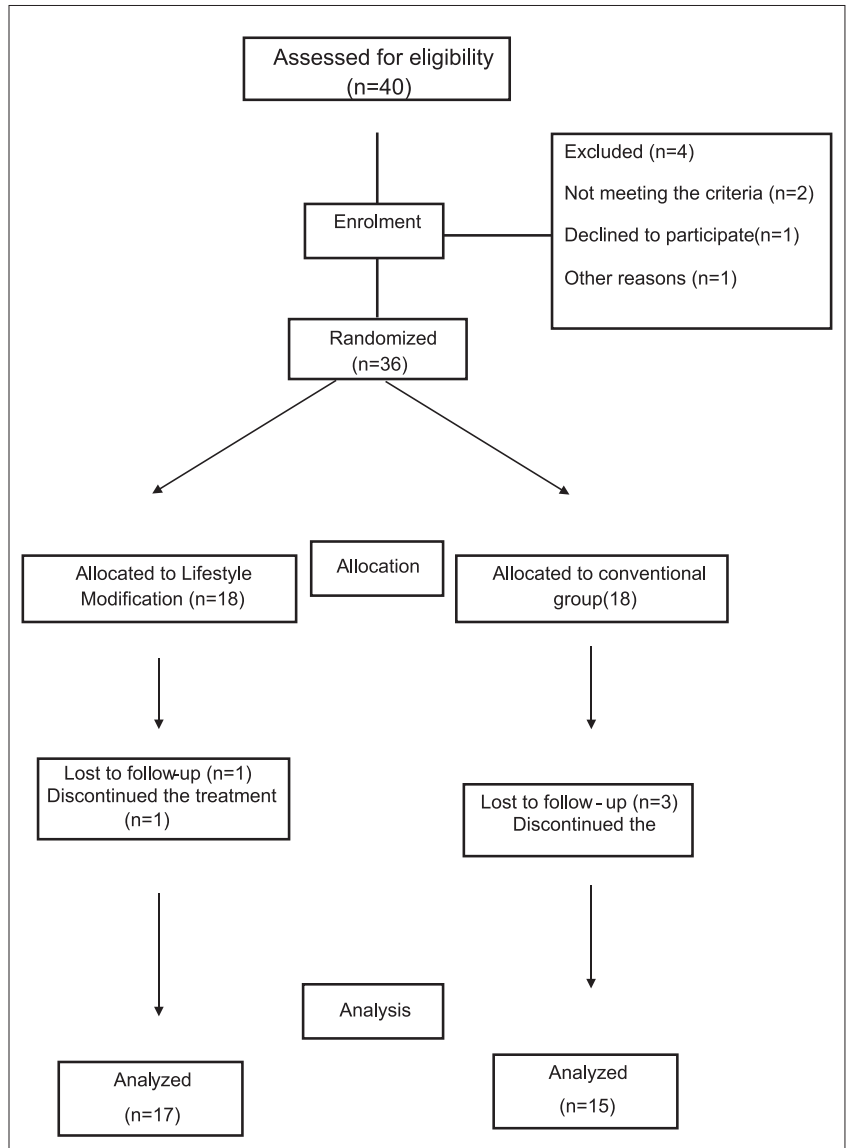

Fig. 1: Flow diagram of allocation of patients, intervention, dropouts, and analysis

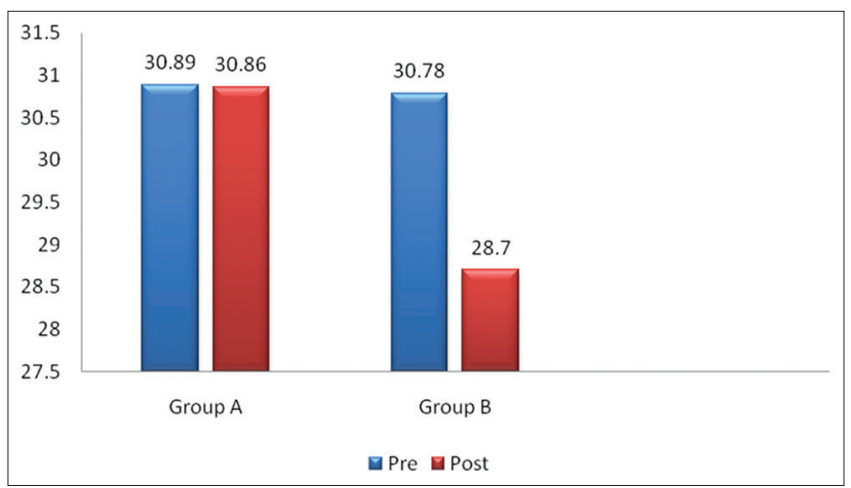

Fig. 2: Comparison of pre and postbody mass index score within groups

as a decrease in glucose transport and decline in muscle glycogen synthesis in response to circulating insulin. PCOS women tend to have an insulin resistant body which makes weight loss as a challenge. Hence, women tend to have marked central obesity with lifestyle modification $\mathrm{W}-\mathrm{H}$ ratio is less altered; however, comparing the pre and posttest, the $\mathrm{W}-\mathrm{H}$ ratio upgraded from good to excellent (W-H ratio norms).

In conventional group, obese women remained obese with a slight increase in BMI because of unrestrained calorie intake and poor behavioral management. In lifestyle modification group with healthy diet with modified exercises and behavioral management to motivate and get into a good body image, obese women has significant weight loss with decreased BMI class from obese to overweight.

PCOS women tend to have pear-shaped body structure. In lifestyle modification group, aerobic exercises targeted the whole-body and

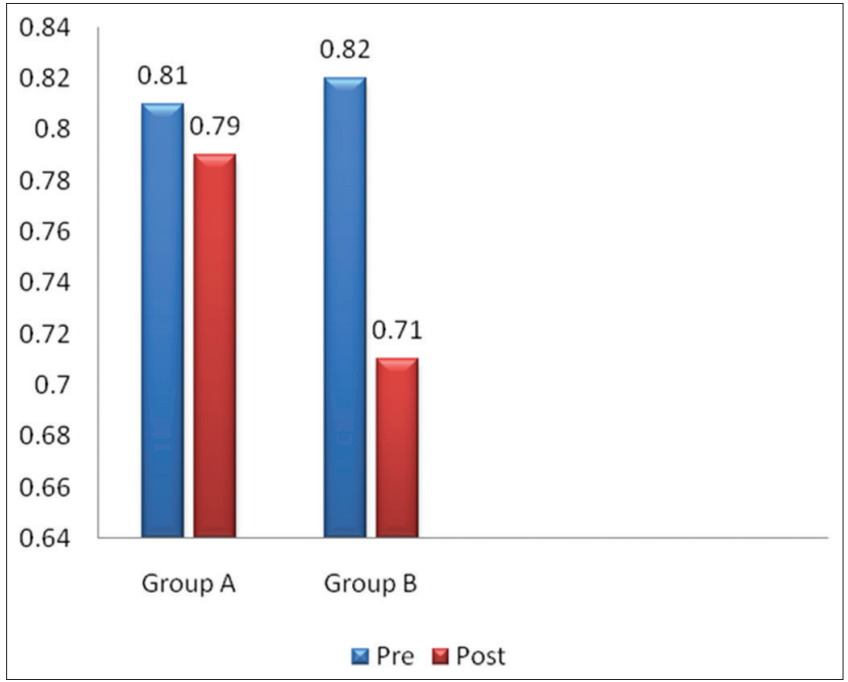

Fig. 3: Comparison of pre and post-Waist-Hip ratio between groups

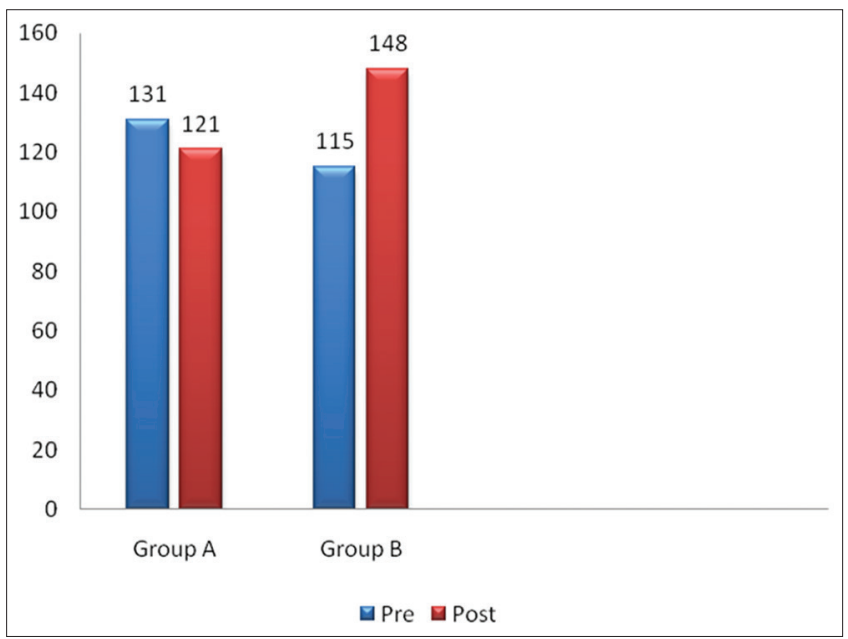

Fig. 4: Comparison of pre and postpolycystic ovarian syndrome questionnaire between groups

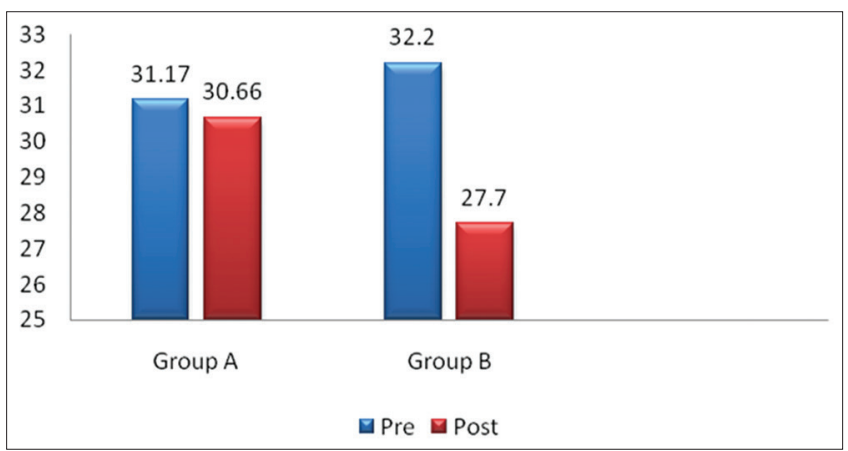

Fig. 5: Comparison of pre and postbody fat percentage between groups

strengthening exercises targeted-specific muscle groups. The body fat percentage decreased as a whole in the body.

Group B was more significant as compared to Group A. Group A was not given behavioral management for stress management and diet. They had no restrictions on calorie intake. Whereas, Group B was given lifestyle modification which included exercises with diet and behavioral management which added plus point in keeping them motivated toward 


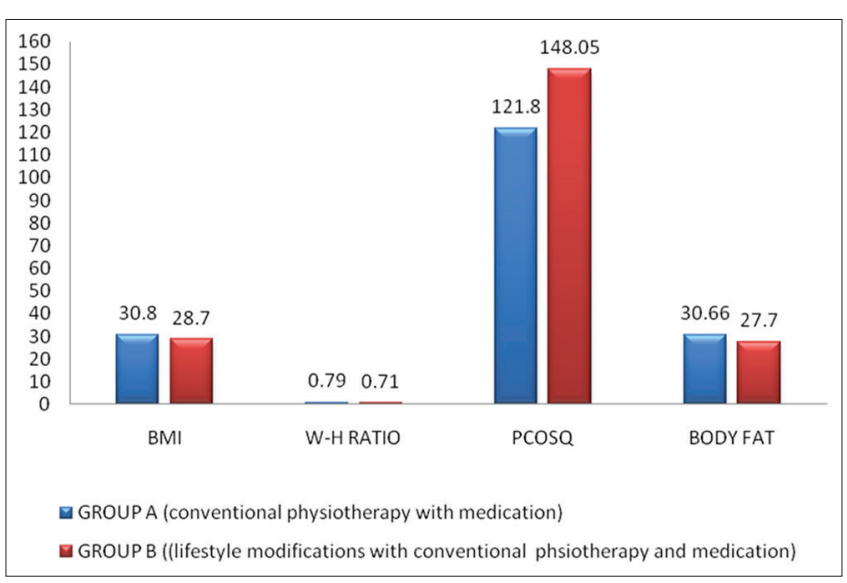

Fig. 6: Comparison between all outcome measures between Group A and $B$

their body image and keep holding their diet by setting different goals and target [Table 5 and Fig. 6].

The conventional group was less effective because no restriction on diet or any calorie intake also behavioral management was not a part of their protocol which showed that conventional lacks as compared to the lifestyle modification. Women felt challenging to continue diet and exercises in their routine, which is further a challenge to keep going with gained weight. Hence, continuing with lifestyle modification is a challenge and should be kept as long-term follow-up, to have good effects.

\section{CONCLUSION}

This study concluded that lifestyle modification for obese PCOS women was effective on weight loss and the quality of life. Apart from exercises with health advice, a structured protocol is very effective in bringing out substantial effect on weight loss outcome measures such as BMI, W-H ratio, and body fat and also improved the quality of life according to PCOSQ. Thereby, we conclude that with medication lifestyle modification should be the first-line treatment in bringing out significant outcome in physical and mental health measures.

\section{ACKNOWLEDGMENT}

The authors would like to acknowledge the guidance and support of Mrudula Mhaske, Akshaya Lade from faculty of physiotherapy, and Dr. Dhiraj Mane for help in statistics.

\section{AUTHOR'S CONTRIBUTION}

Vinaya R. Patil: Conducted literature review for this manuscript, developed introduction section of the manuscript together with the discussion of the study findings, collected data, and analyzed the data. T. Poovishnu Devi: Provided a description of the background information and participated in preparation of the manuscript. Vaishali Krishnat Jagtap: Participated in approval of the manuscript. All the authors read and approved the final manuscript.

\section{CONFLICT OF INTERESTS}

The authors declare that there are no conflicts of interest concerning the content of the present study.

\section{REFERENCES}

1. Moran LJ, Pasquali R, Teede HJ, Hoeger KM, Norman RJ. Treatment of obesity in polycystic ovary syndrome: A position statement of the androgen excess and polycystic ovary syndrome society. Fertil Steril 2009;92:1966-82.

2. Barr S, Hart K, Reeves S, Sharp K, Jeanes YM. Habitual dietary intake, eating pattern and physical activity of women with polycystic ovary syndrome. Eur J Clin Nutr 2011;65:1126-32.

3. Naderpoor N, Shorakae S, de Courten B, Misso ML, Moran LJ, Teede HJ. Metformin and lifestyle modification in polycystic ovarian syndrome. Hum Reprod 2015;21:560-74.

4. Lim SS, Clifton PM, Noakes M, Norman RJ. Obesity management in women with polycystic ovary syndrome. Women's Health 2007;3:73-86.

5. Domecq JP, Prutsky G, Mullan RJ, Hazem A, Sundaresh V, Elamin MB, et al. Lifestyle modification programs in polycystic ovary syndrome. Clin Endocrinol Metab 2013;98:4655-63.

6. Lim AJ, Huang Z, Chua SE, Kramer MS, Yong EL. Sleep duration, exercise, shift work and polycystic ovarian syndrome-related outcomesin a healthy population: A cross-sectional study. PLoS One 2016;11:e 0167048.

7. Jones GL, Benes K, Clark TL, Denham R, Holder MG, Haynes TJ, et al. The polycystic ovary syndrome health-related quality of life questionnaire (PCOSQ). Hum Reprod 2004;19:371-7.

8. Thomson L, Buckley JD, Brinkworth GD. Exercise for the treatment and management of overweight women with polycystic ovary syndrome. Int Assoc Study Obes 2011;12:202-10.

9. Verma A, Kumar S, Dei L, Dhiman K. Management of PCOS: A psychosomatic disorder by yoga practice. Int $J$ Innov Res Dev 2015;4:216-9.

10. Legro RS, Dodson WC, Kunselman AR, Stetter CM, Kris-Etherton PM, Williams NI, et al. Benefit of delayed fertility therapy with preconception weight loss over immediate therapy in obese women with PCOS. J Clin Endocrinol Metab 2016;101:2658-66.

11. Dattatray BM, Devi TP, Jagtap V. Effectiveness of Pilates and strengthening exercise on weight loss and quality of life among Grade II obese adults - Comparative study. Indian J Physiother Occup Ther 2017;11:130-4.

12. Joshi S, Jadhav V, Kadam V. Exotic fruits and vegetable food as nutritional supplement for diabetes, obesity and metabolic diseases. Int J Curr Pharm Res 2018;10:51-5.

13. Jain R, Jain P, Jain P. A review on treatment and prevention of diabetes mellitus. Int J Curr Pharm Res 2016;8:16-8. 